\title{
The influence of inversion domains on surface morphology in GaN grown by molecular beam epitaxy
}

L. T. Romano

T. H. Myers

Follow this and additional works at: https://researchrepository.wvu.edu/faculty_publications

\section{Digital Commons Citation}

Romano, L. T. and Myers, T. H., "The influence of inversion domains on surface morphology in GaN grown by molecular beam epitaxy" (1997). Faculty Scholarship. 373.

https://researchrepository.wvu.edu/faculty_publications/373 


\title{
The influence of inversion domains on surface morphology in GaN grown by molecular beam epitaxy
}

\author{
L. T. Romano a) \\ Xerox Palo Alto Research Center, 3333 Coyote Hill Road, Palo Alto, California 94304 \\ T. H. Myers ${ }^{\text {b) }}$ \\ Department of Physics, West Virginia University, Morgantown, West Virginia 26506
}

(Received 10 September 1997; accepted for publication 10 October 1997)

\begin{abstract}
Growth of $\mathrm{GaN}$ by rf-plasma molecular beam leads to different surface morphologies for nitrogen-rich growth versus gallium-rich growth. Nitrogen-rich growth produces a significant density of pyramidal hillocks while gallium-rich growth results in flat surfaces. Differences in surface morphology were directly linked to the presence of inversion domains which originated in the nucleation layer. Nitrogen-rich growth and growth under atomic hydrogen enhanced the growth rate of inversion domains with respect to the surrounding matrix, while growth under Ga-rich conditions resulted in a more nearly equal growth rate. (c) 1997 American Institute of Physics. [S0003-6951(97)00350-1]
\end{abstract}

Heteroepitaxial GaN layers grown by any technique contain a high dislocation density as well as other classes of defects such as nanopipes, voids and inversion domain boundaries (IDBs). Inversion domains (IDs), consisting of regions of $\mathrm{GaN}$ with the opposite polarity to the primary matrix, have been observed to some extent in $\mathrm{GaN}$ grown by all techniques. ${ }^{1-5}$ However, there is some indication that IDBs are both more common and more stable during growth of $\mathrm{GaN}$ by molecular beam epitaxy (MBE) ${ }^{5,6}$ In this letter, we present the results of a study of IDBs in GaN grown by rf-plasma-assisted MBE detailing how different growth conditions affect the role of IDBs in determining surface morphology.

The presence of IDs have been previously reported for GaN grown by MBE. ${ }^{5,6}$ Samples grown by electron cyclotron resonance (ECR) MBE nucleated under Ga-rich conditions exhibited IDs up to $50 \%$ by volume, ${ }^{5}$ which is significantly larger than that observed in $\mathrm{GaN}$ grown by metalorganic chemical vapor deposition (MOCVD). ${ }^{1-3}$ While films nucleated and grown under nitrogen-rich (N-rich) conditions by ECR-MBE were found to be free of IDBs, significant regions of zincblende material near the interface were observed. $^{5}$ Also, nucleation and growth of $\mathrm{GaN}$ under Garich conditions has resulted in improved structural, electrical and optical properties with smoother surface morphologies when compared to $\mathrm{N}$-rich growth. ${ }^{6-8}$

Atomic force microscopy (AFM) and transmission electron microscopy (TEM) were used to study surface morphology and its relation to microstructure in a series of $\mathrm{GaN}$ layers grown by MBE on (0001) sapphire. Detailed descriptions of the growth procedure and other layer characterizations are given elsewhere. ${ }^{6,9,10}$ In brief, the buffer layers for all samples were grown by heating the substrate to $730^{\circ} \mathrm{C}$ under an atomic hydrogen flux for $\mathbf{2 0} \mathrm{min}$ and then cooling to $630^{\circ} \mathrm{C}$ for the growth of a 200 - $\AA$-thick $\mathrm{GaN}$ buffer layer. ${ }^{6}$ $\mathrm{GaN}$ layers were then grown at a temperature of $730^{\circ} \mathrm{C}$ under various $\mathrm{N}$-rich and $\mathrm{Ga}$-rich conditions, both with and

a)Electronic mail: romano@parc.xerox.com

b)Electronic mail: tmyers@wvu.edu without an atomic $\mathrm{H}$ flux. A standard $\mathrm{Ga}$ source and an $\mathrm{Ox}-$ ford CARS25 rf-plasma nitrogen source were used for the growths. Cross-section TEM (XTEM) studies were performed with a JEOL 3010 microscope operated at $300 \mathrm{kV}$ on samples that were prepared by polishing and then ion milling to electron transparency. TEM images were taken under various diffraction conditions including multiple dark-field imaging with $g= \pm(0002)$ along either the $\langle 1120\rangle$ or $\langle 1010\rangle$ axis in order to reveal inversion domains.' AFM was performed in air using a Digital Instruments Nanoscope II.

As detailed by Yu et al., ${ }^{6}$ AFM measurements indicate smooth surfaces for layers grown under Ga-rich conditions without atomic $\mathrm{H}$, with $\mathrm{rms}$ roughness values $<1 \mathrm{~nm}$. In contrast, samples grown either under $\mathrm{N}$-rich conditions or under an atomic hydrogen flux have a rough surface consisting of pyramidal hillocks. The hillocks have a triangular cross section that is $\sim 0.5 \mu \mathrm{m}$ on a side for $1-\mu \mathrm{m}$-thick samples, and between $150-250 \mathrm{~nm}$ in height. Growth near the $\mathrm{N}$-rich/Ga-rich transition (without atomic $\mathrm{H}$ ) results in smaller size pyramids. The surfaces between the hillocks have been measured by AFM and x-ray reflectivity to have a rms roughness of $1-2 \mathrm{~nm} .^{6,10}$

Figure 1(a) shows a XTEM image of a 1.0- $\mu \mathrm{m}$-thick sample grown under an atomic hydrogen flux with a surface that contains a high density of pyramid-shaped hillocks similar to that observed for all samples grown under nitrogenrich conditions, or with atomic hydrogen. The hillocks are $\sim 100 \mathrm{~nm}$ high and $\sim 200 \mathrm{~nm}$ wide at the base and each contains an ID with a cross section of $\sim 10 \mathrm{~nm}$ as shown in Fig. 1(b). The IDs were found to originate at the film/substrate interface and extend to the film surface with a constant cross sectional area between 5-20 $\mathrm{nm}$ in diameter. The IDBs were along the $\{1010\}$ planes, similar to IDs found in other films grown by $\mathrm{MBE}^{5,6}$ and MOCVD.,

GaN layers grown by MOCVD with a similar surface morphology have been investigated by Daudin et al. ${ }^{2}$ using ion channeling and convergent beam electron diffraction. They found that the IDs associated with pyramids at the surface of their samples were oriented (0001) (or Ga terminated), while the bulk of the matrix was oriented (0001) (or 

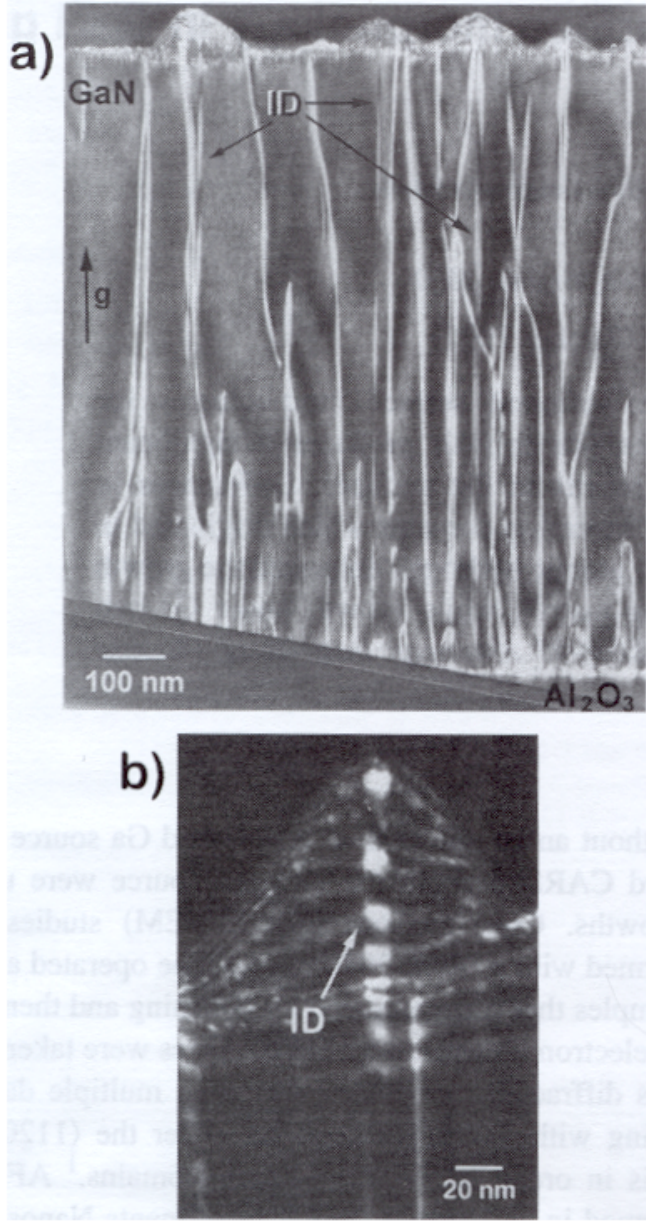

FIG. 1. Multiple dark field images taken with $g=(0002)$ near the $(1120)$ zone axis for a film grown under $\mathrm{Ga}$-rich conditions with atomic hydrogen. (a) Lower magnification of entire film thickness. (b) Higher magnification of pyramid at surface showing the central inversion domain.

$\mathrm{N}$ terminated). In contrast, they were also able to grow "flat" samples which were single phase $(0001)$ and contained no IDs. Hillock formation apparently results from the higher growth rate of the ID located at its midpoint. The resultant strain at the boundary may also enhance the growth rate of the opposite phase and dictate the final surface morphology. Perhaps this non-planar growth region is a source of point defects which degrade the properties of samples grown under $\mathrm{N}$-rich conditions. ${ }^{7}$

We believe that our IDs are oriented (0001) in analogy with the MOCVD results. ${ }^{7}$ This is supported by a recent study by Smith et al. ${ }^{11}$ who found by STM measurements for films grown by rf-plasma MBE using similar techniques that the polarity of the matrix was $\mathrm{N}$ terminated. If our assessment is correct, however, the Ga-terminated surface can have a significantly higher growth rate than a $\mathrm{N}$-terminated surface, in agreement with the speculations of Middleton et al. ${ }^{12}$ One implication is that if the bulk matrix is nucleated to be Ga-terminated and a N-terminated ID is of small cross section, the possibility exists for overgrowth of the ID under $\mathrm{N}$-rich conditions, removing this defect after growth of a sufficient layer thickness.

Samples grown under excess $\mathrm{Ga}$ were found to be free of pyramidal hillocks, even for films containing high densities of IDBs. An example is given in Fig. 2(a) corresponding to a
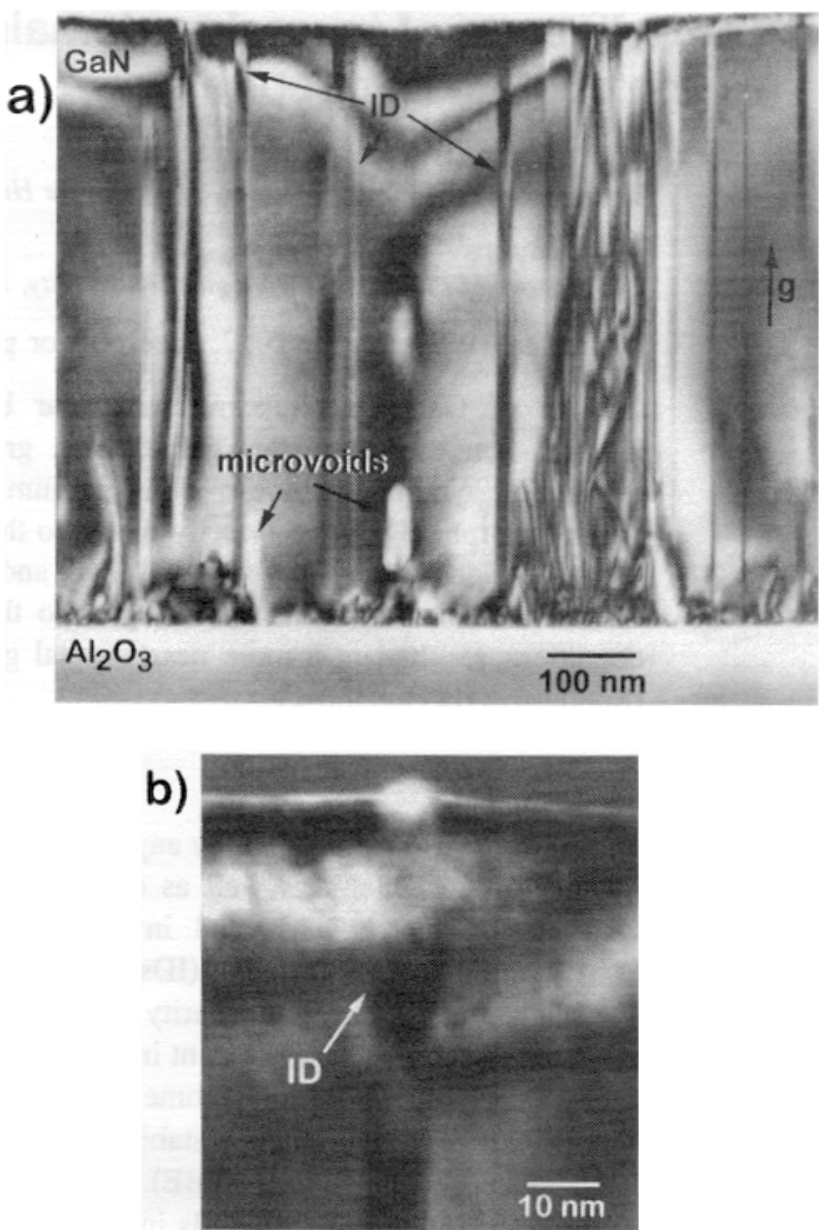

FIG. 2. Multiple dark field images taken with $g=(0002)$ near the $(1120)$ zone axis for a film grown under Ga-rich conditions without atomic hydrogen. (a) Lower magnification of entire film thickness. (b) Higher magnification of an inversion domain at the surface.

sample grown under highly Ga-rich conditions. Again, IDBs were observed to originate at the film/substrate interface and propagate through the growing layer. In sharp contrast to $\mathrm{N}$-rich growth, no significant hillock features were observed at the intersection of the IDB with the surface of the GaN. However, as shown in Fig. 2(b), the surface of the ID is found to be $\mathbf{2} \mathrm{nm}$ higher than the surrounding matrix, indicating a slight enhancement in the growth rate in the region near the ID. This height difference is significantly less than in the film shown in Fig. 1(b). Thus, Ga-rich growth appears to suppress the growth-rate differential between the two different polarities of $\mathrm{GaN}$, directly leading to the observed smoother surface morphologies.

The formation of IDBs is not an intrinsic property of MBE growth of $\mathrm{GaN}$, but is apparently related to nucleation conditions. At least one sample grown in this study had an IDB concentration of $<5 \%$, with an ID cross section of $<20 \mathrm{~nm}$. As shown here, the IDs initiate within the buffer layer and are localized laterally with a relatively small cross section, indicating a localized nucleation site. We obtain different densities of IDBs with what we believe are identical nucleation conditions. This could be due the presence of steps in the sapphire surface as suggested previously, ${ }^{1}$ defects in the substrate surface itself from remnant polishing damage, or possibly related to high energy ions present in the 
nitrogen flux itself. (With respect to the latter, we have measured a small but finite flux of nitrogen ions with energies $>25 \mathrm{eV}$ from our nitrogen source. ${ }^{13}$ )

Nitridation of the sapphire surface prior to growth influences the microstructure and morphology in MOCVD layers. ${ }^{14-16}$ While nitridation is readily accomplished using an ECR plasma source for the active nitrogen, ${ }^{17,18}$ our laboratory and others ${ }^{8,19}$ have not observed any evidence of nitridation with an rf-plasma source for exposures up to one hour. A recent study by Heinlein et al. indicate that the nitridation process is very slow with an rf-plasma source, taking up to $5 \mathrm{~h}$ for the formation of one monolayer. ${ }^{20}$ It may be that nitridation is accomplished by ions with sufficient energy for atomic displacement. The slow nitridation rate would thus be due to the small residual high energy ion content found in an rf-plasma source, while the faster nitridation rate observed with ECR-plasma sources naturally comes about from the significantly higher ion density. The seemingly random nucleation of IDs for layers grown by rf-plasma MBE could thus be accounted by inhomogeneous nitridation on the substrate from residual high energy ions during buffer layer nucleation. Experiments are underway in our laboratory to see how ion content affects sapphire nitridation. It may be that by eliminating ions from our rf-plasma source we can suppress ID formation for what we believe is growth of $(000 \underline{1}) \mathrm{GaN}$.

Alternatively, nitridation of the sapphire may allow the subsequent growth of (0001) GaN. Correct choice of which polar surface to grow can have a marked influence on layer properties, as has been observed in other binary semiconductors. At this time, it is not clear which polarity is best for MBE growth.

Other microstructural features can also be observed in the TEM images that depend on the growth conditions. For the sample grown under $\mathrm{Ga}$-rich conditions with atomic $\mathbf{H}$ [Fig. 1(a)], a higher density of mixed and screw dislocations were found compared to the sample grown under Ga-rich conditions without atomic $\mathrm{H}$ [Fig. 2(a)]. In addition microvoids are present in Fig. 2(a) that are faceted along the $\{1010\}$ and $\{1011\}$ planes. These voids, found to be elongated along the $c$ axis up to $100 \mathrm{~nm}$ in length and $20-50 \mathrm{~nm}$ wide, may be related to $\mathrm{Ga}$ condensation during growth.

In conclusion, the differences in surface morphology observed for different growth conditions could be directly linked to the presence of IDs in our GaN layers. Nitrogenrich growth and growth under atomic hydrogen enhanced the growth rate of IDs with respect to the surrounding matrix, whereas growth under Ga-rich conditions resulted in a more nearly equal growth rate. IDs apparently originated in the initial nucleation layer, and were stable with respect to layer growth. A possible cause of the inversion domains is inhomogeneous nitridation on the sapphire substrate due to a remnant high energy ion content in the nitrogen flux from the rf-plasma source.

The authors would like to thank M. R. Richards-Babb of the Chemistry Department at WVU for performing the AFM measurements. Work at WWVU was supported by DoD/ ONR Grant No. N00014-94-1-1149 and ONR Grant No. N00014-96-1-1008. The work at Xerox was supported by DARPA MDA972-96-3-014.

${ }^{1}$ L. T. Romano, J. E. Northrup, and M. A. O'Keefe, Appl. Phys. Lett. 69 , 2394 (1996).

${ }^{2}$ B. Daudin, J. L. Rouviere, and M. Arley, Appl. Phys. Lett. 69, 2480 (1996).

${ }^{3}$ X. H. Wu, L. M. Brown, D. Kapolonek, S. Keller, B. Keller, S. P. DenBaars, and J. S. Speck, J. Appl. Phys. 80, 3228 (1996).

${ }^{4}$ Z. Liliental-Weber, H. Sohn, N. Newman, and J. Washburn, J. Vac. Sci. Technol. B 13, 1578 (1995)

${ }^{5}$ L. T. Romano, B. S. Krusor, R. Singh, and T. D. Moustakas, J. Electron. Mater. 26, 285 (1997).

${ }^{6}$ Zhonghai Yu, S. L. Buczkowski, N. C. Giles, T. H. Myers, and M. R. Richards-Babb, Appl. Phys. Lett. 69, 2731 (1996); S. L. Buczkowski, Zhonghai Yu, M. R. Richards-Babb, N. C. Giles, T. H. Myers, and L. T. Romano, Mater. Res. Soc. Symp. Proc. 449, 197 (1997).

${ }^{7}$ H. Reichert, R. Averbeck, A. Graber, M. Schienle, U. Straub, and H. Tews, Mater. Res. Soc. Symp. Proc. 449, 149 (1997).

${ }^{8}$ R. Beresford, K. S. Stevens, Q. Cui, A. Schwartzman, and H. Cheng, Mater. Res. Soc. Symp. Proc. 449, 361 (1997).

${ }^{9}$ M. Richards-Babb, S. L. Buczkowski, Zhonghai Yu, and T. H. Myers, Mater. Res. Soc. Symp. Proc. 395, 237 (1996).

${ }^{10}$ D. Lederman, Zhonghai Yu, T. H. Myers, and M. R. Richards-Babb, Appl. Phys. Lett. 71, 368 (1997).

${ }^{11}$ A. R. Smith, R. M. Feenstra, D. W. Grove, J. Neugebauer, and J. E. Northrup (unpublished).

${ }^{12}$ P. G. Middleton, C. Trager-Cowan, A. Mohammed, K. P. O'Donnell, W. Van Der Stricht, I. Moerman, and P. Demeeser, Mater. Res. Soc. Symp. Proc. 449, 471 (1997).

${ }^{13}$ EPI MBE Products Group (St. Paul, MN), Application Note 97-3 (August, 1997).

${ }^{14}$ S. Keller, B. P. Keller, Y.-F. Wu, B. Heying, D. Kapolnek, J. S. Speck, U. K. Mishra, and S. P. DenBaars, Appl. Phys. Lett. 68, 1525 (1996).

${ }^{15}$ N. Grandjean, J. Massies, and M. Leroux, Appl. Phys. Lett. 69, 2071 (1996).

${ }^{16}$ K. Uchida, A. Watanabe, F. Yano, M. Kouguchi, T. Tanaka, and S. Minagawa, J. Appl. Phys. 79, 3487 (1996).

${ }^{17}$ T. D. Moustakas and R. J. Molnar, Mater. Res. Soc. Symp. Proc. 281, 753 (1993).

${ }^{18}$ M. E. Lin, B. N. Sverdlov, and H. Morkoc, J. Appl. Phys. 74, 5038 (1993).

${ }^{19}$ C. Heinlein, J. Grepstad, H. Riechert, and R. Averbeck, Mater. Sci. Eng. B 43, 253 (1997).

${ }^{20}$ C. Heinlein, J. Grepstad, T. Berge, and H. Riechert, Appl. Phys. Lett. 71, 341 (1997). 